ScIDice

\section{International Journal of Dentistry and Oral Science (IJDOS) ISSN: 2377-8075}

\title{
Vitamin k2 - A Review
}

Review Article

Revathi Duraisamy ${ }^{1 *}$, Dhanraj M. Ganapathy ${ }^{2}$, Shanmugam Rajeshkumar ${ }^{3}$

${ }^{1}$ Assistant Professor, Department of Prosthodontics and Implantology, Saveetha Dental College and Hospitals, Saveetha Institute of Medical and Technical Sciences (SIMATS), Saveetha University, 162, Poonamallee High Road, Velappanchavadi, Chennai - 600077.

${ }^{2}$ Professor and Head (Administration), Department of Prosthodontics, Saveetha Dental College and Hospitals, Saveetha Institute of Medical and Technical Science, Saveetha University, 162, Poonamalle high road, Velapanchavadi, Chennai- 600077, Tamil Nadu, India.

${ }^{3}$ Nanobiomedicne Lab, Department of Pharmacology, Saveetha Dental College and Hospital, SIMATS, Saveetha University, Chennai.

\section{Abstract}

\begin{abstract}
Vitamin K1 (phylloquinone), K2 (menaquinone), and K3 (phylloquinone) are the three types of vitamin K. (menadione). Vitamin $\mathrm{K} 2$ is found in both tissue and bacterial products (animal products or fermented foods). Vitamin $\mathrm{k} 2$ has nine chemical variations, with the number of isoprenyl units in their side chains determining the majority of them. The most frequent form of vitamin $\mathrm{k} 2$ in the human diet is the short chain, water soluble menatetrenone, which is generated by bacterial conversion of vitamin $\mathrm{k} 1$, as well as a tissue derivative (MK-4).MK-7, MK-8, and MK-9 are long-chain menaquinones (longer than MK-4) that are more prevalent in fermented foods like natto, a traditional Japanese meal prepared from soya beans fermented with bacillus subtilis var. Anaerobic bacteria in the colon create longer-chain menaquinones (MK-10 to MK-13), but they are poorly absorbed and have minimal physiological impact at this level. The effects of vitamin K2 on overall dentistry are the topic of this review.
\end{abstract}

Keywords: Menaquinone; Fermented Food; Gut Bacteria; Fat Soluble Vitamin; Osteocalcin.

\section{Introduction}

Vitamin $\mathrm{K}$ is found in two physiologically active forms in nature. Vitamin K1, also known as phyllo-quinone (PK), is found in large quantities in leafy green vegetables including cabbage, spinach, and lettuce [1]. Vitamin K2, also known as menaquinone (MK), is a microbially produced version of vitamin K2 [2]. Vitamin K2 is mostly found in fermented foods like cheese and natto (fermented soybeans), but it can also be synthesised by the gut flora. Vitamin $\mathrm{K} 2$ is a fat-soluble vitamin required for healthy teeth and bones. Vitamin K2 is a protein that works in tandem with Vitamin D to transport calcium from soft tissue and the circulation to teeth and bones. Because of the high levels of calcium in saliva, vitamin K2 can also help prevent calculus from accumulating on the outside of upper molars and behind bottom front teeth. Because they are so close to salivary glands in the cheeks and behind the tongue, calculus tends to accumulate in these locations. Vitamin $\mathrm{K} 2$ has also been related to improved brain, kidney, and cancer prevention, as well as blood sugar level stabilization $[1,3]$.
During the post-translational conversion of glutamic acid residues to -carboxyglutamic acid (Gla) in particular proteins, vitamin $\mathrm{K}$ works as a cofactor for the endoplasmic enzyme -glutamylcarboxylase. These proteins are known as vitamin K-dependent proteins, and they contain various blood coagulation and anticoagulation factors that are made in the liver. Osteocalcin (OC), a bone-specific protein produced by osteoblasts, and Matrix Gla Protein (MGP), a multi-organ protein. These vitamin K-dependent Gla proteins, their role in bone metabolism, and their inhibitory influence on arterial calcification have recently received a lot of attention. Vitamin $\mathrm{K}$ intake from a regular diet is currently more than that required for normal blood coagulation in healthy adults, but it is insufficient for additional hepatic tissue requirements $[1,3]$.

When compared to vitamin K1, vitamin K2 has been discovered to be much more effective in bone metabolism. Menaquinone-4 (MK-4) is a high-dose vitamin $\mathrm{K} 2(45 \mathrm{mg} /$ day) used as a thera-

\section{*Corresponding Author:}

Revathi Duraisamy M.D.S

Assistant Professor, Department of Prosthodontics and Implantology, Saveetha Dental College and Hospitals, Saveetha Institute of Medical and Technical Sciences (SIMATS), Saveetha University, 162, Poonamallee High Road, Velappanchavadi, Chennai - 600077.

E-mail: revathid.sdc@saveetha.com

Received: September 12, 2021

Accepted: September 20, 2021

Published: September 21, 2021

Citation: Revathi Duraisamy, Dhanraj M. Ganapathy, Shanmugam Rajeshkumar. Vitamin k2 - A Review. Int J Dentistry Oral Sci. 2021;8(9):4388-4392. doi: http://dx.doi.org/10.19070/2377-8075-21000893

Copyright: Revathi Duraisamy M.D.S. ${ }^{2021}$. This is an open-access article distributed under the terms of the Creative Commons Attribution License, which permits unrestricted use, distribution and reproduction in any medium, provided the original author and source are credited. 
peutic therapy for osteoporosis in Japan. Vitamin K2's main effect on osteoporosis is to prevent bone fractures by enhancing bone quality rather than increasing bone mineral density. Another vitamin $\mathrm{K} 2$ homolog, menaquinone-7 (MK-7) derived from $\mathrm{Ba}-$ cillus subtilis natto, has recently attracted attention. Vitamin K2 is required because it starts the calcium transport pathway from the bloodstream to the bones, particularly the teeth. Calcium builds up in the bloodstream due to a lack of Vitamin K2 in our diets, obstructing blood flow in the arteries and putting you at risk of heart disease. Fortunately, there are a few excellent sources of Vitamin K2 that can be included in one's diet. Grass-fed beef, liver, free-range eggs, and Gouda cheese are all examples. At nutritional doses, this has been demonstrated to be particularly effective in carboxylation of osteocalcin [4].

\section{Non-dietary sources of menaquinones}

The gut microbiota or bacteria present in diet may produce bacterially manufactured menaquinones that contribute to human vitamin K2 requirements. Bacteroides and Bifidobacteria are the most important genera of gut flora in humans. Menaquinone can only be synthesised by Bacteroides.Bacteroides produces two primary forms: MK10 and MK11. MK6 produced by Eubacterium lentum, MK7 produced by Veillonella, and MK8 produced by Enterobacter were also discovered in intestinal flora isolates [5]. The distal colon contains the majority of menaquinones, but the terminal ileum, which contains menaquinone-producing bacteria and bile salts required for menaquinone solubilization, is the most promising site of absorption [6]. As a result, despite the fact that intestinal microflora produce significant amounts of menaquinones, bacterial menaquinone has limited bioavailability, and diet is the primary source of functionally accessible vitamin $\mathrm{K} 2$ [6, 7]. Intestinal menaquinones do not compensate for a short-term drop in dietary vitamin $\mathrm{K}$ consumption, according to recent research [4].

Foods high in Vitamin K2 (Dietary sources of menaquinones) [2]

Consuming regular servings of the following high $\mathrm{K} 2$ foods as part of a nutrient-dense whole food diet, will increase vitamin $\mathrm{k} 2$ levels in blood.

\section{- Organic grass-fed butter}

- Organic unhomogenised grass-fed full cream milk

- Organic grass-fed meats

- Vintage cheddar cheese - aged over 18 months and depends on cheese culture

- $\quad$ Fatty fish including cold water (North Atlantic) wild caught salmon, sardines and mackerel

- Naturally-rich free range egg yolks

- Goose liver

- Fermented soy natto - a Japanese food product

\section{Dietary recommendations for menaquinone}

Depending on country, sex, and age, the recommendations for vitamin $\mathrm{K}$ range from 50 to $120 \mu \mathrm{g}$ per day for adults 19 years and older $[8,9]$.

\section{Pharmacokinetics of menaquinones}

Unlike phylloquinone, which is largely stored in the liver for clotting factor synthesis, menaquinones are released into the circulation, where they are incorporated into low-density lipoproteins and delivered to Gla protein carboxylation sites in bone and arteries. Longer half-lives, up to several days for long chain menaquinones, compared to phylloquinone, which generally disappears from the bloodstream after 8 hours, promote absorption.This prolonged postprandial presence in the bloodstream results in a more stable circulating level of vitamin $\mathrm{K} 2$ and, as a result, a longer availability of these long chain menaquinones for extrahepatic tissue uptake [10]. Even though there is some indication that medium chain menaquinones like MK7 are effectively absorbed than short (MK4) or long chain menaquinones (MK8 and MK9) [10, 11], human data on the bioavailability, absorption, and kinetics of K2 vitamins from food is restricted to MK7 and MK9, and has not been systematically tested for all menaquinones to date [12].

\section{Vitamin K2 in calcium homeostasis}

Vitamin K2, a lipid soluble vitamin, which plays a pivotal role in blood coagulation and calcium homeostasis [13]. It was initially thought that Vit K2 is essential for the activation of several proteins, including prothrombin, proconvertin etc. responsible for blood coagulation. However, later studies found that Vit K2 was responsible for the activation of several proteins (osteocalcin , matrix Gla protein (MGP), growth arrest-specific protein 6, Gla-rich protein and several others) associated with bone remodeling also $[14,15]$. For instance, bone remodeling is mainly mediated cells such as osteoblasts, osteocytes and osteoclasts. Among these, osteoblasts synthesize osteocalcin, a non-collagenous, Vit $\mathrm{K} 2$ dependent, and a calcium binding protein in the bone microenvironment. Osteocalcin is responsible for calcium uptake from systemic circulation and binds it to the bone mass. Osteocalcin exists as i.e. carboxylated and undercarboxylated circulatory forms [16]. Carboxylated form of osteocalcin is responsible for calcium-hydroxyapatite binding and precipitation, which allow mineralization of bone matrix. After this mineralization process carboxylated osteocalcin remains confined in the bone matrix, and it is released as undercarboxylated form into circulation upon bone degradation. Thus, carboxylated and undercarboxylated forms of osteocalcin are considered biomarkers of bone turnover in healthy and osteoporotic individuals [17]. Thus osteocalcin regulates bone mineralization by binding into hydroxyl appetite, a mineral component of bone matrix. This process makes bone stronger and reduces the fracture vulnerability. Vit K2 is an essential factor for the Matrix Gla Protein (MGP) and osteocalcin activation and it's binding to the calcium. Mechanistically, Vit K2 controls oxidative stress through nuclear steroid and xenobiotic receptors and increases the osteoblasts proliferation and their activity via MGP and Wnt/ $\beta$-catenin signaling pathway. Vitamin K2 inhibits bone resorption via osteoprotegerin activation. Vitamin $\mathrm{K} 2$ induce osteoblastogenesis and reportedly regulating osteoclastogenesis mainly via the activation of NF- $x \mathrm{~B}$ (RANK) ligand (RANKL)/osteoclast differentiation factor axis as compared to the osteoprotegerin/osteoclast inhibitory factor axis [18]. Vit K2 also regulates osteoclasts and controls the bone resorption [19]. Similarly, Vit K2 supplementation was shown to increase the bone mineral density in experimental animals and also to promote in vitro osteoblast differentiation and mineralization and inhibit osteoclast resorption activity [20].

Vit $\mathrm{K} 2$ is reported to inhibit bone resorbing factors such as pros- 
Figure 1. Role of vitamin $\mathrm{K}$ in bone remodeling. MGP, matrix gla protein; NFkB, nuclear factor kb; OC, osteocalcin; cOC, carboxylated osteocalcin; Hap, hydroxyapatite; Ca, calcium ions; RANKL, NF- $\varkappa$ B (RANK) ligand (RANKL).

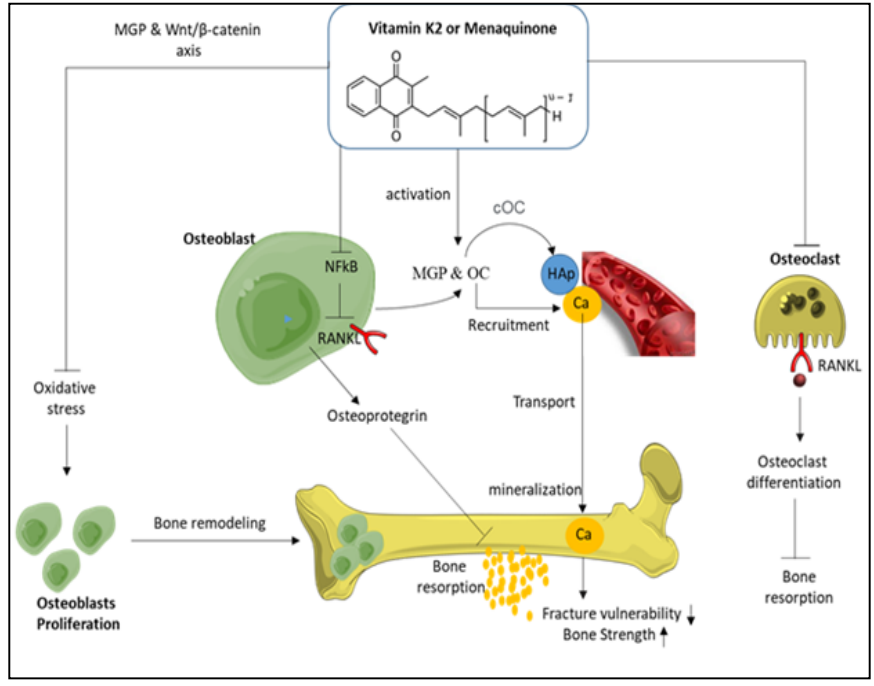

taglandin $\mathrm{E} 2$, interlukin $1 \alpha$, and 1, 25(OH) $2 \mathrm{D} 3$ induced bone resorption [21]. Vitamin K2 $(100 \mu \mathrm{g} / \mathrm{g})$ treatment was shown to inhibit the apoptosis and aortic calcification induced by warfarin. Vitamin K2 treatment significantly decreased alkaline phosphatase activity and calcium deposition and promoted the apoptosis markers via activation of growth arrest-specific protein 6/ AXL pathway in calcification model of rats [14, 22]. Experimental studies have shown that Vit K2 could control bone resorption by i) reducing the nuclear factor $\mathrm{k} \beta$ expression in osteoclast by ii) inhibiting the receptor activator of RANKL expression by osteoclasts [23] iii) inhibiting the osteoclastic differentiation induced by RANKL [24] and iv) inducing the osteoprotegerin action.

The bone remodeling role of Vit $\mathrm{K}$ is depicted in Figure 1.

\section{Vitamin $\mathrm{k} 2$ and bone metabolism}

Vitamin K2 (MK menaquinone) belongs to a broad range of fatsoluble chemicals that appear to be involved in a variety of biological processes. Because it contributes to the structural integrity of osteocalcin (OC), the principal non-collagenous protein normally found in bone matrix, vitamin K2 has lately been identified as safe and effective in the treatment of bone loss. Low vitamin K2 intake has been associated with bone loss and increased fracture risk in both men and women, according to several studies. Nowadays, vitamin $\mathrm{K} 2$ supplementation is thought to be an important way to improve the relationship between calcium and vitamin $\mathrm{D}$, both of which have a well-known role in bone health. Vit K2, on the other hand, can be taken alone or in combination with other medications to protect bone quality and strength after menopause and/or in individuals with secondary osteoporosis [25].

Osteoporosis is a public health issue linked to a higher risk of bone fractures and vascular calcification. Vitamin K, despite being understudied, has particular benefits in various areas. Phylloquinone (vitamin K1) and menaquinone (vitamin K2) are the two major forms of vitamin K. (vitamin K2). The activity of vitamin K2 in bones and arteries was studied in depth in this study. Vitamin K2 has been demonstrated to promote bone formation by boosting alkaline phosphatase, insulin-like growth factor-1, growth differentiation factor-15, and stanniocalcin 2 levels, as well as stimulating osteoblast differentiation and carboxylation of osteocalcin. Vitamin K2 also lowers osteoclast differentiation by raising osteoprotegerin and decreasing the receptor activator of nuclear factor kappa-B ligand, which diminishes the pro-apoptotic proteins Fas and Bax in osteoblasts. Vitamin K2 inhibits the apoptosis of vascular smooth muscle cells by increasing growth arrest-specific gene 6 , and reduces the transdifferentiation of vascular smooth muscle cells to osteoblasts in blood vessels by carboxylation of matrix Gla protein and Gla rich protein. It also inhibits the apoptosis of vascular smooth muscle cells by increasing growth arrest-specific gene 6. In human research, the most widely used dosage of vitamin $\mathrm{K} 2$ is $45 \mathrm{mg}$ /day, and its use could be a promising method for improving bone and vascular health, particularly in osteoporotic postmenopausal women [26].

In an experiment conducted [25, 27-35], they discovered that by consistently consuming adequate amounts of vitamins $\mathrm{K} 2, \mathrm{D}$, and calcium from childhood, humans' peak bone mass can be increased, and that this diet will slow the rate of bone mass loss, thereby limiting bone fractures caused by osteoporosis.

The European Food Safety Authority (EFSA) determined that an acceptable intake of vitamin $\mathrm{K} 1$ - the most common form of vitamin $\mathrm{K}$ in foods - for all adults, including pregnant and lactating women, might be about 70 micrograms per day.The concentrations used in bone clinical research usually correspond to 45 $\mathrm{mg} /$ day of vitK2 as MK-4 or MK-7, although vitK2 is currently available in a variety of formulations in conjunction with vitD3 and $\mathrm{Ca}$ at a $45 \mathrm{mcg}$ dosage [27]. Supplementing with vitamin K2 appears to be effective in maintaining bone health and preventing osteoporosis.

\section{Vitamin $\mathrm{k} 2$ and Dental caries}

When disease bacteria use sugars and starches from dietary items to produce acid, dental caries develop. It attacks the tooth enamel first, causing holes in it. If not addressed promptly, it can result in pain, infection, and tooth loss. Inorganic and organic substances will be demineralized and disintegrated from the tooth material. Saliva provides nourishment to dental tissues from the exterior of the tooth. Minerals, enzymes, and buffering agents are present 
in these fluids, which provide sustenance. Saliva also aids in the maintenance of appropriate mineralization of the enamel of the teeth through buffering demineralization caused by acid-induced mineral breakdown and delivering nutrients for remineralization [36].

It is unknown how saliva is linked to vitamin $\mathrm{K} 2$, however it has been linked to its $\mathrm{pH}$-buffering inorganic phosphate content, which has been linked to lower lactobacilli levels in the oral cavity. Some cheeses have been claimed to have anticariogenic qualities, owing to the presence of fermented bacteria that create significant levels of vitamin $\mathrm{K} 2$. Cheese has been categorised as anticariogenic, although milk is non-cariogenic. Because of its mineral makeup, milk may have a local effect on the caries process. Cheeses, otherwise, are fermented with bacteria, resulting in much more K2 [37].

The beneficial effect of cheese then could be systemic as a source of $\mathrm{K} 2$ rather than local as a non-acid producing food or mineral provider. Alternately or additionally, there is a possibility that K2 may be absorbed across the oral mucous membranes. Recent studies have effectively applied ubiquinone as a topical to suppress periodontal inflammatory reactions due to oxidative stress In a nutshell, $\mathrm{K} 2$ has an effect on the outside of the tooth via influencing saliva content. Dietary nutrients are the best source of primary prevention or maintenance of mineralization. If the content of saliva can be changed to generate a pro-remineralization environment, secondary prevention or re-mineralization success will be significantly determined. In terms of prevention of dental caries, optimum nutrition with fat soluble vitamins like K2 plays a far more significant role than the traditional dental recommendation to simply eat less sugar to minimize oral bacterial acids. Dental disease will be recognized as another inflammation related degenerative lifestyle disease like cardiovascular disease, osteoporosis and diabetes [25, 27-35].

\section{Vitamin k2 and Periodontal health}

Vitamin D3 and vitamin K2 have been shown to have a synergistic impact in preventing bone loss. In rats with experimentally induced periodontitis, the effects of vitamin D3 and vitamin K2 supplementation in combination with conventional periodontal therapy (scaling and root planning $[\mathrm{SRP}]$ ) on gingival interleukin (IL)-1band IL-10, serum bone alkaline phosphatase (B-ALP) and tartrate-resistant acid phosphatase 5b(TRAP-5b), and calcium and alveolar bone levels supplementation with vitamin D3 in conjunction with conventional periodontal therapy reduced gingival IL-10 levels significantly suggests that vitamin D3 and vitamin k2 may have protective functions on gingival inflammation[38]. Matsunaga et al. reported that vitamins D and K2 combined had synergistic effects in the reduction of bone loss in ovariectomized rats [39].

\section{Vitamin $\mathrm{k} 2$ and Implant survival}

Surgical difficulties, bone quality and quantity, and host-related factors, such as patients' nutritional state, all affect dental implant osseointegration. Many micronutrients may influence various alveolar bone parameters, such as alveolar bone repair following tooth extraction, and hence play a vital role in dental implant osseointegration.
Manufacturers have created a variety of procedures, both chemical (acid-etching) and mechanical (grit-blasting) or a combination of the two, to improve the contact area between the living bone and the implant and therefore boost osseointegration. Addition (titanium plasma spray), subtraction (acid-etching, grit-blasting), or a combination of procedures can be used to create rough titanium implant surfaces. For surface treatments, grit-blasting is a standard physical procedure.A projection of silica, hydroxyapatite, alumina, and $\mathrm{TiO} 2$ particles with sizes ranging from 25 to $75 \mathrm{~m}$ is used. Temperature, pressure, kind, and size of blasting particles are only a few of the variables that might affect the end output. The implants' alumina remnants and blasting-modified surface energy promote cell adherence but inhibit cell growth [40].

Many commercial procedures have been established to produce a thin coat in order to improve the roughness of the surface (solgel deposition, sputtering coating processes, or ion beam-assisted deposition).

In dentistry, nanoparticles as technological accelerators have promise. The inclusion of nanoparticles on the implant surface can alter the topography as well as the surface chemistry, resulting in unique implant specifications. The osteoconductive substance hydroxyapatite is used in titanium-based implants. In dental implantation, nano hydroxyapatite gives more surface area and reactivity [41].

Vitamin K2, on the other hand, is critical since it is the vitamin that initiates the process of calcium transportation from the circulation to the bones, particularly the teeth [42]. The main goal of implant surface modifications is to improve clinical performance in places where there is a lack of bone quantity or quality, as well as to stimulate bone growth to allow implant placement in locations where there isn't enough residual alveolar ridge. The level of BIC in a dental implant is a key factor in its long-term success. The goal of treatment has become to maximise the BIC, which is aided by implant surface roughness and osseointegration [43]. As a result, it is sensible to assume that titanium dental implants layered with nanohydroxyapatite and Vitamin K2, vitamin D, and chitosan may have better biocompatibility and osseointegration properties. As a result, it is of interest to prepare titanium dental implants coated with nanohydroxyapatite and Vitamin K2 and study their biocompatibility and osseointegration.

\section{Conclusion}

Vitamin K's major impacts on bone health are to maintain and improve bone quality rather than to enhance bone mineral density. The mechanisms could include activating osteocalcin, enhancing collagen matrix, and influencing osteoblast and osteoclast differentiation. MK-7 has the highest activity and bioavailability among the vitamin $\mathrm{K}$ homologs in humans. As a result, MK-7 is thought to enhance bone health at dietary doses. In order to improve dental implant survival and success rates, a larger intervention trial for $\mathrm{MK}-7$ is required.

\section{References}

[1]. Walther B, Chollet M. Menaquinones, bacteria, and foods: vitamin K2 in the diet. Vitamin K2-Vital for Health and Wellbeing. 2017 Mar 22:63-82.

[2]. Yasin M, Butt MS, Zeb A. Vitamin K2 rich food products. Vitamin K2: Vital for Health and Wellbeing. 2017 Mar 22:35. 
[3]. Shearer MJ. Vitamin K. The Lancet. 1995;345(8944):229-34.

[4]. Urano A, Hotta M, Ohwada R, Araki M. Vitamin K deficiency evaluated by serum levels of undercarboxylated osteocalcin in patients with anorexia nervosa with bone loss. Clin Nutr. 2015 Jun;34(3):443-8. Pubmed PMID: 24909585

[5]. Ramotar K, Conly JM, Chubb H, Louie TJ. Production of menaquinones by intestinal anaerobes. J Infect Dis. 1984 Aug;150(2):213-8. Pubmed PMID: 6470528.

[6]. Conly JM. Assay of menaquinones in bacterial cultures, stool samples, and intestinal contents. Methods Enzymol. 1997;282:457-66. Pubmed PMID: 9330310.

[7]. Binkley NC, Suttie JW. Vitamin K nutrition and osteoporosis. J Nutr. 1995 Jul;125(7):1812-21. Pubmed PMID: 7616295.

[8]. Ferland G. The discovery of vitamin K and its clinical applications. Ann Nutr Metab. 2012;61(3):213-8. Pubmed PMID: 23183291.

[9]. Shearer MJ, Fu X, Booth SL. Vitamin K nutrition, metabolism, and requirements: current concepts and future research. Adv Nutr. $2012 \mathrm{Mar}$ 1;3(2):182-95. Pubmed PMID: 22516726.

[10]. Vermeer C. Vitamin K: the effect on health beyond coagulation - an overview. Food Nutr Res. 2012;56. Pubmed PMID: 22489224.

[11]. Schurgers LJ, Vermeer C. Determination of phylloquinone and menaquinones in food. Effect of food matrix on circulating vitamin K concentrations. Haemostasis. 2000 Nov-Dec;30(6):298-307. doi: 10.1159/000054147. PMID: 11356998.

[12]. Beulens JW, Booth SL, van den Heuvel EG, Stoecklin E, Baka A, Vermeer C. The role of menaquinones (vitamin $\mathrm{K}_{2}$ ) in human health. Br J Nutr. 2013 Oct;110(8):1357-68. Pubmed PMID: 23590754.

[13]. Khalil Z, Alam B, Akbari AR, Sharma H. The Medical Benefits of Vitamin K2 on Calcium-Related Disorders. Nutrients. 2021 Feb 21;13(2):691. Pubmed PMID: 33670005.

[14]. Gordeladze J, editor. Vitamin K2: Vital for Health and Wellbeing. BoDBooks on Demand; 2017 Mar 22.

[15]. Wen L, Chen J, Duan L, Li S. Vitamin K dependent proteins involved in bone and cardiovascular health (Review). Mol Med Rep. 2018 Jul;18(1):315. Pubmed PMID: 29749440.

[16]. Ferron M, Wei J, Yoshizawa T, Del Fattore A, DePinho RA, Teti A, Ducy P, Karsenty G. Insulin signaling in osteoblasts integrates bone remodeling and energy metabolism. Cell. 2010 Jul 23;142(2):296-308. Pubmed PMID: 20655470.

[17]. Eastell R, Pigott T, Gossiel F, Naylor KE, Walsh JS, Peel NFA. DIAGNOSIS OF ENDOCRINE DISEASE: Bone turnover markers: are they clinically useful? Eur J Endocrinol. 2018 Jan;178(1):R19-R31. Pubmed PMID: 29046326

[18]. Koshihara Y, Hoshi K, Okawara R, Ishibashi H, Yamamoto S. Vitamin $\mathrm{K}$ stimulates osteoblastogenesis and inhibits osteoclastogenesis in human bone marrow cell culture. J Endocrinol. 2003 Mar;176(3):339-48. Pubmed PMID: 12630919.

[19]. Myneni VD, Mezey E. Regulation of bone remodeling by vitamin K2. Oral Dis. 2017 Nov;23(8):1021-1028. Pubmed PMID: 27976475.

[20]. Boulier A, Schwarz J, Lespesailles E, Baniel A, Tomé D, Blais A. Combination of micellar casein with calcium and vitamins D2 and K2 improves bone status of ovariectomized mice. Osteoporos Int. 2016 Oct;27(10):3103-12. Pubmed PMID: 27222105

[21]. Akbari S, Rasouli-Ghahroudi AA. Vitamin K and Bone Metabolism: A Review of the Latest Evidence in Preclinical Studies. Biomed Res Int. 2018 Jun 27;2018:4629383. Pubmed PMID: 30050932.

[22]. Jiang X, Tao H, Qiu C, Ma X, Li S, Guo X, Lv A, Li H. Vitamin K2 regression aortic calcification induced by warfarin via Gas6/Axl survival pathway in rats. Eur J Pharmacol. 2016 Sep 5;786:10-18. Pubmed PMID: 27212383.

[23]. Yamaguchi M, Weitzmann MN. Vitamin K2 stimulates osteoblastogenesis and suppresses osteoclastogenesis by suppressing NF- $\triangle \mathrm{B}$ activation. Int J Mol Med. 2011 Jan;27(1):3-14. Pubmed PMID: 21072493.

[24]. Wu WJ, Kim MS, Ahn BY. The inhibitory effect of vitamin K on RANKL- induced osteoclast differentiation and bone resorption. Food Funct. 2015 Oct;6(10):3351-8. Pubmed PMID: 26267519.

[25]. Capozzi A, Scambia G, Migliaccio S, Lello S. Role of vitamin K2 in bone metabolism: a point of view and a short reappraisal of the literature. Gynecol Endocrinol. 2020 Apr;36(4):285-288. Pubmed PMID: 31711322.

[26]. Villa JKD, Diaz MAN, Pizziolo VR, Martino HSD. Effect of vitamin K in bone metabolism and vascular calcification: A review of mechanisms of action and evidences. Crit Rev Food Sci Nutr. 2017 Dec 12;57(18):39593970. Pubmed PMID: 27437760.

[27]. Capozzi A, Scambia G, Lello S. Calcium, vitamin D, vitamin K2, and magnesium supplementation and skeletal health. Maturitas. 2020 Oct;140:5563. Pubmed PMID: 32972636.

[28]. Frandsen NE, Gordeladze JO. Vitamin K2 and Bone Health. InVitamin K2Vital for Health and Wellbeing 2017 Mar 22 (pp. 101-123). IntechOpen.

[29]. Møller M, Tonstad S, Bathen T, Gordeladze JO. The Impact of Vitamin K2 on Energy Metabolism. Vitamin K2: Vital for Health and Wellbeing. 2017 Mar 22:83.

[30]. Sato T. Vitamin K2 and bone quality. Vitam Trace Elem S. 2013;6:21670390

[31]. Hirano J, Ishii Y. Effects of vitamin K2, vitamin D, and calcium on the bone metabolism of rats in the growth phase. J Orthop Sci. 2002;7(3):364-9. Pubmed PMID: 12077663.

[32]. Ozuru R, Sugimoto T, Yamaguchi T, Chihara K. Time-dependent effects of vitamin K2 (menatetrenone) on bone metabolism in postmenopausal women. Endocr J. 2002 Jun;49(3):363-70. Pubmed PMID: 12201222.

[33]. Ozaki D, Kubota R, Maeno T, Abdelhakim M, Hitosugi N. Association between gut microbiota, bone metabolism, and fracture risk in postmenopausal Japanese women. Osteoporos Int. 2021 Jan;32(1):145-156. Pubmed PMID: 33241467

[34]. Bartl R, Bartl C. The Osteoporosis Manual: Prevention, Diagnosis and Management. Springer; 2019 Feb 6.

[35]. Southward K. A hypothetical role for vitamin K2 in the endocrine and exocrine aspects of dental caries. Med Hypotheses. 2015 Mar;84(3):276-80. Pubmed PMID: 25636605.

[36]. Lyu XH, Wang K, Zhang LL. [Research progress of the relationship between Candida albicans and dental caries]. Zhonghua Kou Qiang Yi Xue Za Zhi. 2021 May 9;56(5):491-496. Chinese. Pubmed PMID: 33904286.

[37]. Rheaume-Bleue K. Vitamin K2 and the calcium paradox: how a little-known vitamin could save your life. John Wiley \& Sons; 2011 Nov 7.

[38]. Uchiyama S, Kimura M, Masuda Y, Yamaguchi M. Oral Administration of Cal K2 Containing Menaquinone-4 (Vitamin K2) Enhances Serum r-Carboxylated Osteocalcin and Biochemical Components in the Femoral Tissues of Rats. Journal of health science. 2006;52(6):825-30.

[39]. Matsunaga K, Enjoji M, Karube Y, Takata J. Enhanced Intracellular Delivery and Improved Antitumor Efficacy of Menaquinone-4. InVitamin K2-Vital for Health and Wellbeing 2017 Mar 22. IntechOpen.

[40]. Hintze V, Bierbaum S, Scharnweber D. Implant surface modifications and new development in surface coatings. InDental Implants and Bone Grafts 2020 Jan 1 (pp. 89-124). Woodhead Publishing.

[41]. Kang JI, Choe HC, Son MK. Nano/Micro-Sized Morphologies of Hydroxyapatite Coatings Containing $\mathrm{Mn}$ and $\mathrm{Si}$ on an Oxidized Ti-6Al4V Alloy Surface for Dental Implants. J Nanosci Nanotechnol. 2021 Jul 1;21(7):3701-3706. Pubmed PMID: 33715677.

[42]. Verma VK, Hemlata V, Kusum G. A comparative study of efficacy of calcium+ vitamin D3 and calcium+ vitamin D3+ vitamin K2 in prevention of osteoporosis in postmenopausal osteopenic women who are adopting a sedentary pattern of life style. Journal of Evolution of Medical and Dental Sciences. 2013 May 20;2(20):3620-7.

[43]. Annunziata M, Guida L. The Effect of Titanium Surface Modifications on Dental Implant Osseointegration. Front Oral Biol. 2015;17:62-77. Pubmed PMID: 26201277. 